\title{
Therapeutic effect of intense pulsed light with optimal pulse technology on meibomian gland dysfunction with and without ocular Demodex infestation
}

\author{
Yanan Huo ${ }^{1 \#}$, Yanping $\mathrm{Mo}^{2 \#}$, Yaying Wu ${ }^{1}$, Fei Fang ${ }^{3}$, Xiuming Jin ${ }^{1}$ \\ ${ }^{1}$ Department of Ophthalmology, The Second Affiliated Hospital of Zhejiang University School of Medicine, Hangzhou, China; ${ }^{2}$ Department of \\ Ophthalmology, Huzhou Third Municipal Hospital, Huzhou, China; ${ }^{3}$ Department of Ophthalmology, Wuning County People's Hospital, Jiujiang, \\ China \\ Contributions: (I) Conception and design: X Jin, Y Huo, Y Mo; (II) Administrative support: X Jin; (III) Provision of study materials or patients: Y Huo; \\ (IV) Collection and assembly of data: Y Huo, Y Mo; (V) Data analysis and interpretation: Y Mo, Y Wu, F Fang; (VI) Manuscript writing: All authors; \\ (VII) Final version of the manuscript: All authors. \\ "These authors contributed equally to this work. \\ Correspondence to: Xiuming Jin. Department of Ophthalmology, the Second Affiliated Hospital of Zhejiang University School of Medicine, 88\# Jiefang \\ Road, Hangzhou 310009, China. Email: lzyjxm@zju.edu.cn.
}

Background: To evaluate the therapeutic effect of intense pulsed light (IPL) therapy with optimal pulse technology (OPT) on meibomian gland dysfunction (MGD) with and without ocular Demodex infestation.

Methods: This prospective study included 150 patients with MGD who were divided into Demodex-infested (DI) and non-infested (control) groups according to Demodex counts determined by epilating eyelashes to evaluate. Each patient underwent three OPT treatment sessions at 3-week intervals. Best corrected visual acuity (BCVA), intraocular pressure (IOP), Demodex counts, Ocular Surface Disease Index (OSDI) score, conjunctival congestion, tear meniscus height (TMH), tear breakup time (TBUT), Schirmer I test (SIT), corneal and conjunctival fluorescein staining scores, meibomian gland (MG) macrostructure, lid margin abnormality, MG expressibility, and meibum quality were assessed before the first treatment as baseline and at 1, 2, and 3 months (M1, M2 and M3, respectively) after treatment.

Results: The incidence of Demodex infestation was $59.15 \%(84 / 142)$ at baseline and the final Demodex eradication rate at M3 was $83.3 \%$ (70/84). Corneal staining and conjunctival congestion showed slower improvement in the DI group than in the control group at $\mathrm{M} 1$ and $\mathrm{M} 2(\mathrm{P}<0.05)$, but was significantly improved at $\mathrm{M} 3$ than that at with baseline $(\mathrm{P}<0.01)$. Both the groups showed significant improvement in OSDI, conjunctival congestion, TBUT, corneal and conjunctival staining, MG dropout, lid margin abnormality, MG expressibility, and meibum quality at $\mathrm{M} 3(\mathrm{P}<0.05)$. No differences were observed for BCVA, IOP, SIT, and TMH before and after treatment in both the groups $(\mathrm{P}>0.05)$.

Conclusions: This new-generation IPL with OPT treatment results in greater improvement in MG expressibility and meibum quality in MGD patients with Demodex infestation than in those without, but not for corneal staining.

Keywords: Demodex; intense pulsed light; optimal pulse technology, meibomian gland dysfunction

Submitted Feb 19, 2020. Accepted for publication Nov 13, 2020.

doi: 10.21037/atm-20-1745

View this article at: http://dx.doi.org/10.21037/atm-20-1745 


\section{Introduction}

Demodex spp. are host-adapted mites of mammals including humans. Of the many Demodex species, only D. folliculorum and $D$. brevis can parasitize the human eye. D. folliculorum is most commonly found in eyelash follicles, whereas D. brevis usually colonizes the sebaceous and meibomian glands (MGs) (1). Demodex infestation and the waste produced by the mite can block follicles and glands, and trigger the inflammatory response in the anterior and posterior lid margins. Demodex infestation is closely related to MG dysfunction (MGD), which is the most common cause of evaporative dry eye, and its symptoms include ocular surface burning and irritation, redness, pain, fluctuating visual acuity, unexplained keratitis, superficial corneal vascularization, or nodular corneal scarring $(2,3)$. The discomfort caused by these symptoms, combined with the ineffectiveness of conventional treatment, can severely affect the quality of life of patients; therefore, the development of alternative treatment options is underway.

Intense pulsed light (IPL) has been widely used in the cosmetic industry since 1996 for facial dermatological conditions. It has been medically certified to improve subjective symptoms and gland function in patients with MGD (4,5). Moreover, the combination of IPL and MG expression (MGX) is known to improve dry eye symptoms and MG function (6). In a previous multicenter, randomized controlled trial, we showed that the combination of IPL and MGX ameliorated symptoms and assisted in tear film recovery (7).

In 2002, Prieto et al. found that Demodex organisms appeared to be coagulated after IPL treatment for facial cutaneous disease (8), which led to our interest in evaluating IPL as a potential therapy for treating ocular Demodex infestation. However, the effect of IPL treatment on Demodex blepharitis has not been well studied. To date, only a few studies have reported using IPL for successfully treating ocular demodicosis $(9,10)$.

The fifth-generation IPL with optimal pulse technology (OPT) is regarded as the latest technology. To evaluate the efficacy and safety of OPT + MGX combined therapy in patients with and without ocular Demodex infestation, we conducted a pioneering, prospective, consecutive, controlled study for assessing OPT as a therapy for treating ocular demodicosis. This report has been prepared in accordance with the STROBE reporting checklist (available at http:// dx.doi.org/10.21037/atm-20-1745).

\section{Methods}

\section{Subjects}

This study was conducted in compliance with the Declaration of Helsinki (as revised in 2013) for research involving human participants and was approved by The Second Affiliated Hospital of Zhejiang University School of Medicine Ethics Committee (No. 2019-283). Trial registration: ChiCTR1900025925, registered 14 September 2019. We obtained written informed consent from all the patients before their enrollment.

The inclusion criteria were as follows: age $>18$ years, diagnosis of MGD (> stage 1); and including ocular symptoms, such as plugged gland orifices, lid margins vascularity, lid margins irregularity, and decreased meibum quality and quantity. Skin type was determined using the Fitzpatrick scale. Only patients with skin type 4 or lower were treated with OPT (11). Patients who had received implants beneath the treatment area or had acute solar dermatitis, allergic disease, eye surgery in $<1$ month, or any topical or systemic diseases that could affect the results were excluded from the study.

\section{Experiment design}

This was a prospective, consecutive, cohort study. We enrolled 150 patients with MGD (52 men and 98 women) who presented with dry eye syndrome to The Second Affiliated Hospital of Zhejiang University School of Medicine from October 2019 to April 2020. The patients were divided into Demodex-infestated (DI) and non-infested groups after they underwent Demodex examination as reported previously (12). Briefly, three eyelashes from each eyelid of both the eyes were epilated and examined under a light microscope. Patients with Demodex counts of not less than three per three eyelashes were considered Demodex positive.

Each patient underwent three OPT-MGX treatment sessions at 3 -week intervals and three follow-up examinations at 1, 2, and 3 months (M1, M2, and M3, respectively) after the final treatment. Best corrected visual acuity (BCVA) and intraocular pressure (IOP) were recorded for evaluating OPT-MGX safety. The Ocular Surface Disease Index (OSDI) score, conjunctival congestion, tear breakup time (TBUT), Schirmer I test (SIT), tear meniscus height (TMH), corneal and conjunctival fluorescence staining, MG macrostructure, lid margin abnormalities, 
MG expressibility, and meibum quality were determined for evaluating treatment efficacy on the day before treatment at baseline and at each follow-up. Two doctors individually performed the OPT treatment and measurements for all the patients. Only one eye of each patient was analyzed in this study. In patients with or without binocular infestation, only the right eye was analyzed, whereas in patients with single-eye infestation, the infested eye was analyzed and the patient was included in the DI group.

\section{OPT treatment}

We used the M22 IPL system with OPT (Lumenis, Tel Aviv, Israel) and the treatments were administered using the proprietary AOPT mode setting. Energy parameters were determined based on Fitzpatrick skin type and patient tolerance and comfort (range, $15-17 \mathrm{~J} / \mathrm{cm}^{2}$ ). IPL treatment was administered twice from the right preauricular area, across the cheeks and nose and to the left preauricular area, with the treatment area reaching up to the interior boundary of the eye shields. Immediately after OPT treatment, MGX was performed on both the upper and lower eyelids of each eye using an Aritia Meibomian Gland Compressor (Katena Products Inc., Denville, NJ, USA). During the procedure, all the patients received $0.4 \%$ oxybuprocaine hydrochloride eye drops (Santen Pharmaceutical Co., Ltd, Osaka, Japan) to minimize pain. During the study, all patients received sodium hyaluronate eye drops (Santen Pharmaceutical Co., Ltd.) four times a day.

\section{Evaluation of dry eye symptoms}

The severity of ocular surface symptoms was determined using the OSDI questionnaire, which consisted of 12 questions regarding the presence and frequency of symptoms associated with the ocular surface. The total OSDI score (0-100 points) was calculated using the sum score of all completed questions. The final scores were classified according to the severity of the symptoms as follows: score of 0-12 (no symptom); 13-22 (mild); 23-32 (moderate), and 33-100 (severe).

Tear production was assessed using the SIT by inserting a sterile dry strip (Jingming New Technological Development Co. Ltd, Tianjing, China) into the lateral canthus of the lower eyelid, away from the cornea, for $5 \mathrm{~min}$. The length of the strip that became wet because of the absorbed tears was then measured to determine the function of tear glands.

$\mathrm{TMH}$ and conjunctival congestion were measured using
Keratograph 5M (Oculus ${ }^{\circledR}$, Wetzlar, Germany).

Tear film stability was evaluated by determining the TBUT, which was measured by instilling fluorescein into the lower conjunctival sac using a moist fluorescein strip (Jingming New Technological Development Co, Ltd.). The patients were then required to blink several times to ensure adequate coating of the dye on the cornea. The tear film was observed under a slit-lamp biomicroscope with a cobalt blue filter. The test was repeated three times, and the average TBUT was calculated.

Corneal and conjunctival fluorescein staining was performed using the same fluorescein strip used for measuring TBUT. The cornea was divided into five areas (central, superior, temporal, nasal, and inferior). For each area, the corneal severity was graded on a $0-3$ scale. Conjunctiva staining was scored between 0 and 3 .

\section{Evaluation of MG morphology}

MG dropouts were observed using Keratograph 5M (Oculus) and scored on a scale of 0-3: 0, no dropouts; 1 , $<1 / 3$ dropouts; 2, 1/3-2/3 dropouts; and 3, >2/3 dropouts.

Lid margin abnormalities were scored on a scale of $0-4$ based on the following four criteria: irregular lid margins, vascular engorgement, plugging of MG orifices, and shift in the mucocutaneous junction.

MG expressibility was evaluated by applying digital pressure on both the upper and lower tarsi. We divided the entire lid range into three areas (nasal, central, and temporal sides), and observed five glands in each area amounting to a total of 15 glands. The degree of expressibility was graded on a scale of $0-3$ for each area according to the number of glands expressible: 0, all glands; 1, 3-4 glands; 2, 1-2 glands; and 3 , no glands (total score range, $0-9$ ).

To evaluate meibum quality, eight glands in the center of both the upper and lower lids were evaluated on a scale of $0-3$ for each gland: 0 , clear; 1 , cloudy; 2 cloudy with debris (granular); and 3 , thick, toothpaste-like (total score range, $0-24$ ).

\section{Safety assessments}

BCVA, IOP, and lens opacity were observed at baseline and at each follow-up. The skin area around the eye was also examined for depigmentation, blistering, swelling, and redness.

\section{Statistical analyses}

All statistical analyses were performed using SPSS 20.0 
(SPSS Inc., Chicago, IL, USA). A minimum sample size of 45 patients per group was calculated on the basis of an assumed mean CFS difference of 1 between the DI and control groups for a two-tailed test at an alpha level of 0.05 and a power of $90 \%$. Assuming a drop-off rate of $20 \%$, the sample size was determined to be 57 patients per arm. The data of patients who discontinued the study prematurely were excluded from analysis. Numerical data are presented as the means \pm standard deviations (SD). Variables were tested for normality using the KolmogorovSmirnov test. The patients' demographic characteristics were compared using analysis of variance or the Chisquare test. The date of the two groups were compared using the Mann-Whitney $U$ test. Logarithm of the minimum angle of resolution (LogMAR) BCVA, Demodex counts, OSDI, TMH, conjunctival congestion, corneal and conjunctival fluorescence staining, lid abnormality, and MG expressibility, meibum quality were also analyzed using the Mann-Whitney $U$ test. IOP was analyzed using independent-sample $t$-tests. $\mathrm{P}$ values of $<0.05$ were considered statistically significant.

\section{Results}

In this prospective study, we recruited 150 patients with MGD (greater than stage 1) from The Second Affiliated Hospital of Zhejiang University School of Medicine. In the DI group, three patients withdrew from the study prematurely: one patient withdrew before the third treatment because of a car accident, while two patients missed M2 and were considered lost to follow-up. In the control group, five patients withdrew from the study prematurely: one patient withdrew after receiving the first treatment because of pregnancy, one patient withdrew before $\mathrm{M} 1$ because of pneumonia, and three patients missed M3 and were considered lost to follow-up. Their data were excluded from analysis. A flowchart that summarizes the progress through the various phases of the study (enrollment, withdrawal, treatments, and follow-ups) is given in Figure 1.

A total of 142 patients completed all treatments and followup sessions. Eighty-four patients comprised the DI group (29 men and 55 women, mean age, $43.9 \pm 13.74$ years; range, 21-70 years); whereas the control group consisted of 58 patients (20 men and 38 women; mean age, $38.72 \pm 14.22$ years, range, 18-67 years). No significant differences were observed in the demographic data between the two groups at baseline (Table 1).
The DI group exhibited more MGD-related ocular irritation and lid margin inflammation. OSDI, lid margin abnormality, and upper lid MG expressibility were significantly different between the Demodex-positive (DI group) and Demodex-negative (control) eyes at baseline (all, $\mathrm{P}<0.05$ ).

\section{Demodex infestation before and after OPT-MGX treatment}

The incidence of ocular Demodex infestation in patients with MGD was $59.15 \%(84 / 142)$. In the DI group, the mean Demodex count at baseline was $6.74 \pm 3.09$ and significantly decreased at follow-up $(3.18 \pm 3.56$ at M1, $1.76 \pm 1.85$ at M2, and $1.23 \pm 1.56$ at $\mathrm{M} 3$; all, $\mathrm{P}<0.01)$. The Demodex eradication rate was $59.5 \%(50 / 84)$ at $\mathrm{M} 1,66.67 \%(56 / 84)$ at $\mathrm{M} 2$, and $83.3 \%(70 / 84)$ at $\mathrm{M} 3$ (all, $\mathrm{P}<0.05)$. Table 2 shows the general characteristics of the eyes in the DI and control groups before and after OPT-MGX treatment.

\section{Improvement in dry eyesymptoms after OPT-MGX treatment}

The DI group had a significantly higher OSDI score than the control group at baseline $(\mathrm{P}<0.05)$, which decreased immediately after treatment at $\mathrm{M} 1$; the score decreased steadily from M1 to M2 $(\mathrm{P}<0.01)$ and stabilized from M2 to $\mathrm{M} 3(\mathrm{P}=0.676)$. The control group also had a significantly different OSDI score before and after OPT-MGX treatment. The symptoms were relieved from severe to moderated in both the groups; at $\mathrm{M} 3$, the final scores were similar between the two groups $(\mathrm{P}=0.684)$ (Figure 2).

Compared with baseline, TBUT, and conjunctival staining significantly improved immediately at $\mathrm{M} 1$ in both the groups (all, $\mathrm{P}<0.01$ ).

The DI group showed slower changes in corneal staining and conjunctival congestion. In the DI group, corneal staining decreased significantly at M2 $(\mathrm{P}<0.01)$, while in the control group, it decreased earlier at $\mathrm{M} 1(\mathrm{P}<0.01)$; no difference was observed between the two groups at M3 $(\mathrm{P}=0.322)$ (Figure 3).

In the DI group, conjunctival congestion decreased significantly at $\mathrm{M} 3(\mathrm{P}<0.01)$, while in the control groups, it decreased earlier at M2 $(\mathrm{P}<0.05)$. The TMH and SIT of both the groups showed no change over the study period (all, $\mathrm{P}>0.05$ ).

\section{Comparison of clinical results after OPT-MGX treatment}

The MG dropouts at baseline were no different between the 


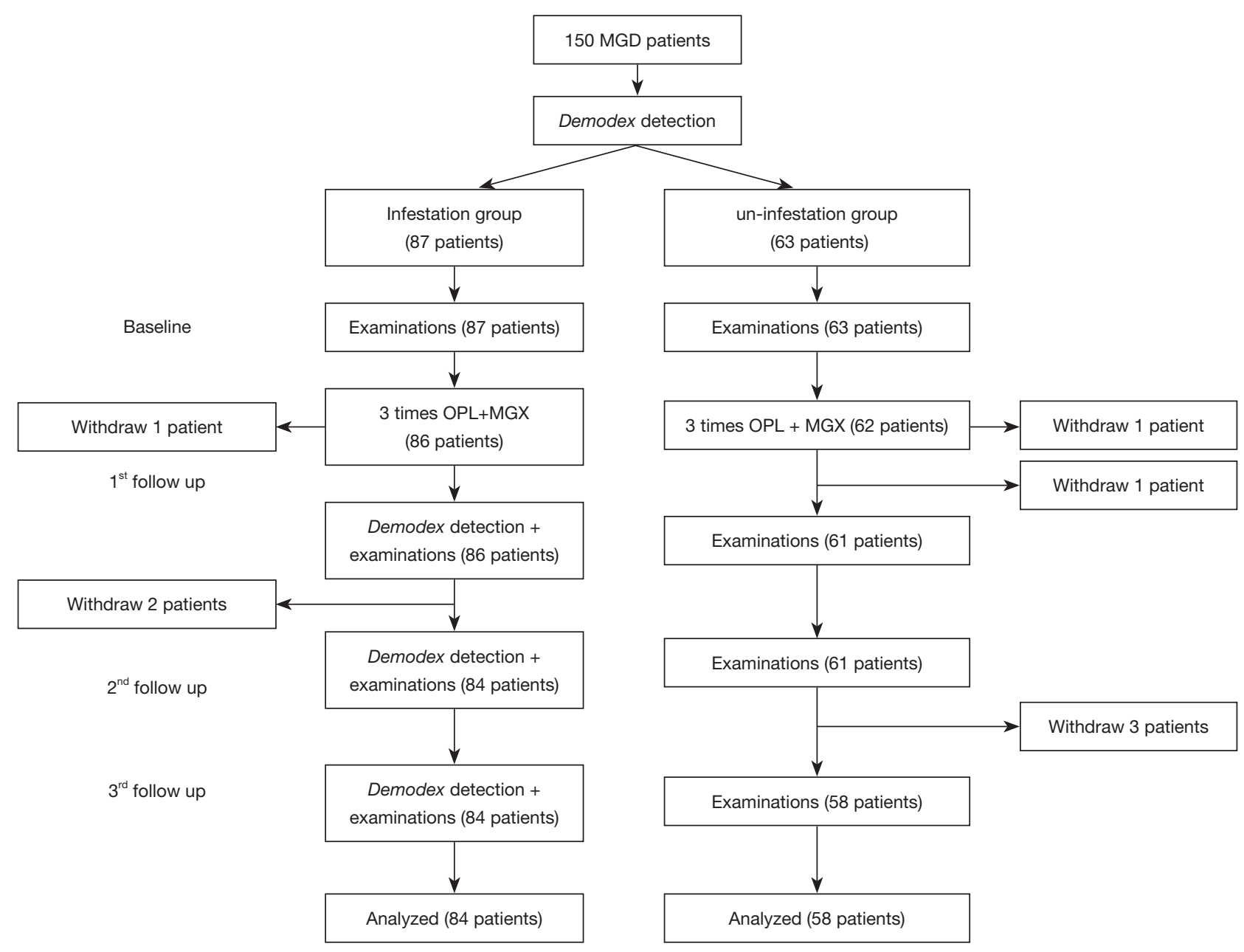

Figure 1 Treatment and follow-up protocol for OPT + MGX. OPT, optimal pulse technology; MGX, meibomian gland expression.

Table 1 Demographic data in DI group and control group (mean \pm SD)

\begin{tabular}{lccc}
\hline & $D I(n=84)$ & Control $(\mathrm{n}=58)$ & $P$ value \\
\hline Age (years) & $43.90 \pm 13.74$ & $38.72 \pm 14.22$ & 0.196 \\
Sex (male/female) & $29 / 55$ & $23 / 35$ & 0.533 \\
\hline
\end{tabular}

DI, Demodex infestation; SD, standard deviation.

two groups, and showed a significant improvement after OPTMGX treatment. At M3, patients in the DI group recovered better than those in the control group $(\mathrm{P}=0.023)$ (Figure 4).

At baseline, the DI group had more serious lid margin abnormality than the control group $(\mathrm{P}<0.01)$; both the groups showed significant improvement after OPT-MGX treatment $(\mathrm{P}<0.05)$. However, the Demodex-negative eyes showed better manifestation than the Demodex-positive eyes at $\mathrm{M} 1$ and $\mathrm{M} 2$ (both, $\mathrm{P}<0.05)$ (Figure 5).
The DI group had more serious upper lid MG expressibility than the control group $(\mathrm{P}<0.05)$. Both upper/lower lid MG expressibility and meibum quality improved significantly after OPT-MGX treatment at M1 (all, $\mathrm{P}<0.05$ ), and the treatment outcome in both the groups was similar at M3.

\section{Discussion}

MGD is a chronic, diffuse abnormality of the MGs 


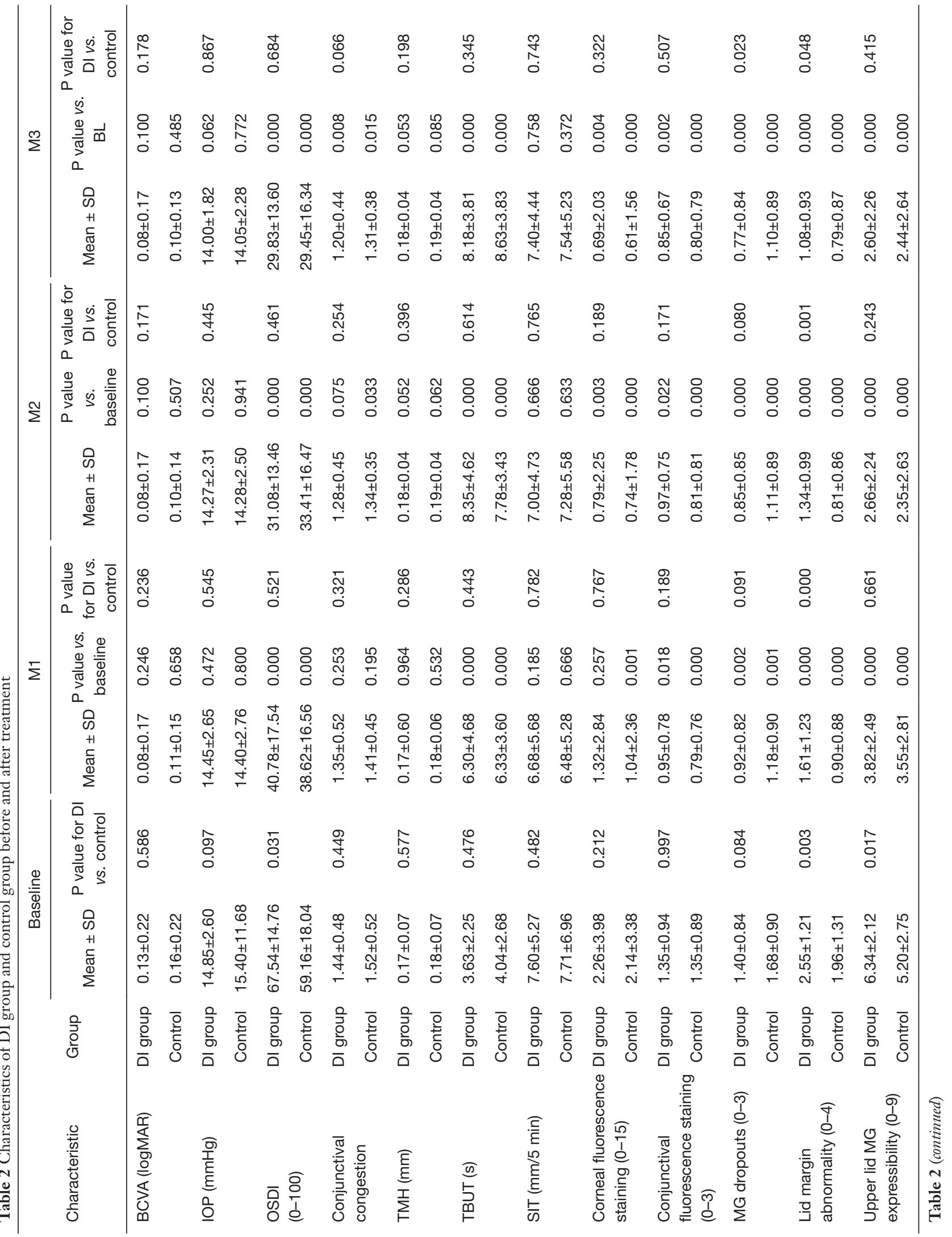




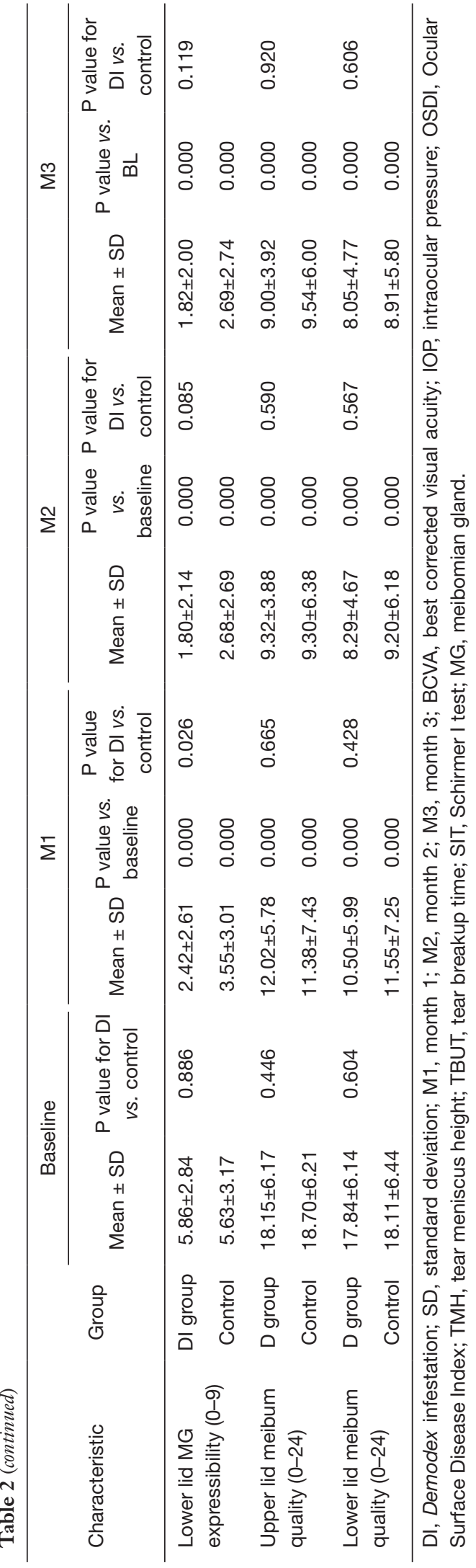

characterized by terminal duct obstruction and qualitative/ quantitative changes in glandular secretion (13). It is the most common cause of evaporative dry eye and affects approximately $45-70 \%$ of the population in Asia $(14,15)$. The reported incidence of Demodex infestation in MGD patients worldwide is approximately $60-86.4 \%$ (16-18). In the present study, $59.15 \%$ of patients with MGD were infested with Demodex. We compared the therapeutic effect of OPT on MGD patients with and without Demodex infestation, and found that OPT was effective in alleviating MGD and eradicating ocular Demodex infestation. Furthermore, comparison of the data obtained for the two groups before and after treatment showed that before OPT treatment, Demodex-positive eyes showed more serious manifestation on OSDI, lid margin abnormality, and MG expressibility on the upper lid. The Demodex-positive eyes also showed less efficiency on lid margin abnormality, and slower recovery of corneal staining and conjunctival congestion at M1 and M2 compared with the Demodex-negative eyes.

Several traditional treatment methods, such as tea tree oil (TTO) (19-21), 1\% yellow mercury ointment (22), 2\% topical metronidazole gel (23), 1\% acaricide permethrin (24), and daily lid scrubbing and cleaning (25), can be used for eradicating ocular Demodex infestation. The life cycle of mites is approximately 14-18 days from the egg to the larval stage, followed by 5 days in the adult stage. Thus, prevention of mating is important. Therefore, these treatment methods have to be used daily for 1-3 months. Most patients find it difficult to comply for such a long treatment period. These traditional treatments are commonly considered unsatisfactory in the clinic setting (26). In 2002, Prieto et al. reported that IPL could cause marked damage inflicted to Demodex organisms found on the facial skin (8). The ocular and facial Demodex mites have the same origin, which led to our interest in evaluating IPL as a potential therapy for ocular Demodex infestation. Although it is widely accepted that IPL can improve meibum quality and expressibility, its therapeutic effect on ocular Demodex infestation was unclear.

Very few studies have evaluated the effect of IPL for treating ocular Demodex infestation. Recently, Zhang et al. compared the treatment effect of IPL and 5\% TTO for ocular demodicosis (9). They found that the eradication rate was greater and more reliable in the IPL group (20/20, $100 \%)$ than in the TTO group (15/20, $75 \%)$. In another retrospective study, Cheng et al. counted the number of Demodex mites before and 1 week after IPL treatment by in vivo confocal microscopy (IVCM) (10). The medical 


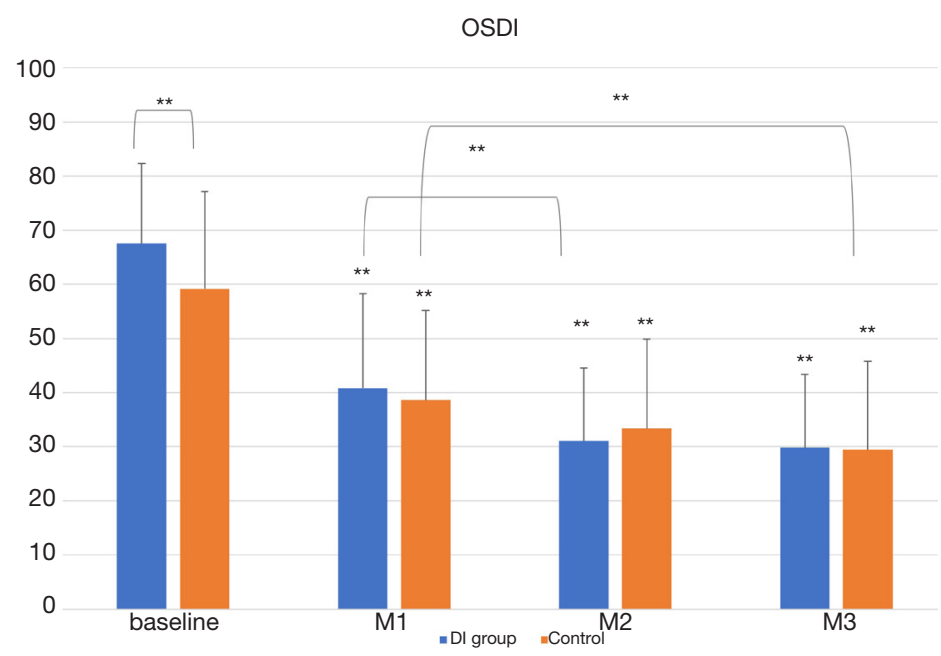

Figure 2 ODSI score at baseline, M1, M2 and M3 in DI group (blue) and control group (orange). The DI group had a higher OSDI score than the control group at baseline $(\mathrm{P}<0.01)$. In the DI group, the OSDI score was better at $\mathrm{M} 3$ than at $\mathrm{M} 2(\mathrm{P}<0.01)$. In the control group, OSDI score was better at M3 than at M1 $(\mathrm{P}<0.01)$. The OSDI score in both groups was improved significantly from baseline at any visit (all $\mathrm{P}<0.01) .{ }^{*} \mathrm{P}<0.01$. OSDI, Ocular Surface Disease Index; M1, month 1; M2, month 2; M3, month 3; DI, Demodex infestation.

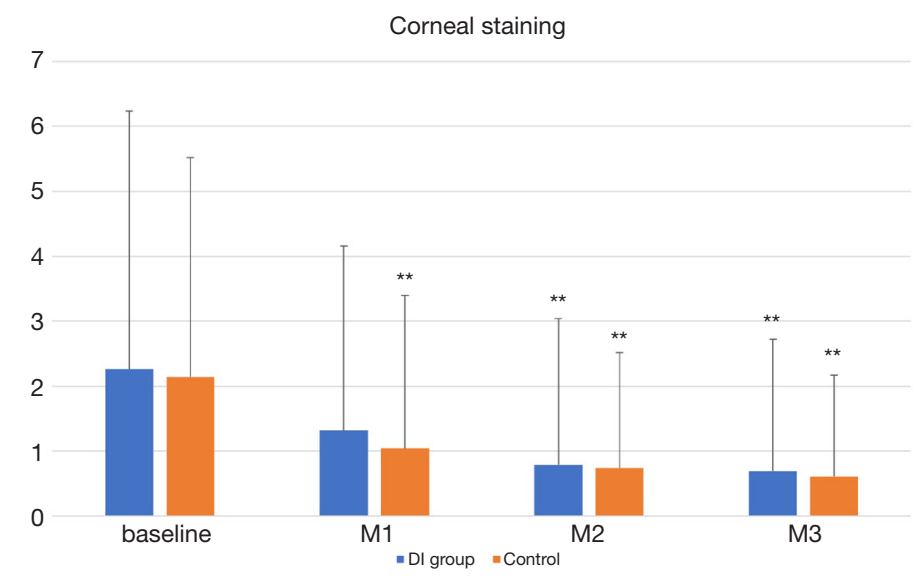

Figure 3 Corneal staining at baseline, M1, M2, and M3 in the DI group and control group. Corneal staining in the DI group decreased at M2 ( $\mathrm{P}<0.01)$. The control eye was different from baseline at any visit (all, $\mathrm{P}<0.01)$. ${ }^{* *} \mathrm{P}<0.01$. M1, month 1; M2, month 2; M3, month 3; DI, Demodex infestation.

records of 25 patients (49 eyes) showed that the Demodex eradication rate was $20 \%(8 / 40)$ in the upper lid margin and $34.15 \%(14 / 14)$ in the lower lid margin. The significantly different results between these two studies may be because of the following reasons. First, both the studies used different detection methods. Zhang et al. counted mites via epilated eyelashes under a light microscope, while Cheng et al. counted mites by IVCM. Demodex infestation is classically diagnosed by analyzing depilated eyelashes under a light microscope. However, the most recent studies have showed that the Demodex mite and larvae inside the lash follicles could be better detected by IVCM (27). Second, Cheng et al. analyzed the eradication rate of the upper and lower lids separately instead of analyzing them simultaneously per person. Third, they only reviewed the data 1 week after treatment and the follow-up period was too short for observing the cumulative effect of IPL treatment. In the study conducted by Zhang et al., the eradication rate was $55 \%(11 / 20) 1$ month after IPL treatment and had reached $100 \%$ by 3 months. In the 


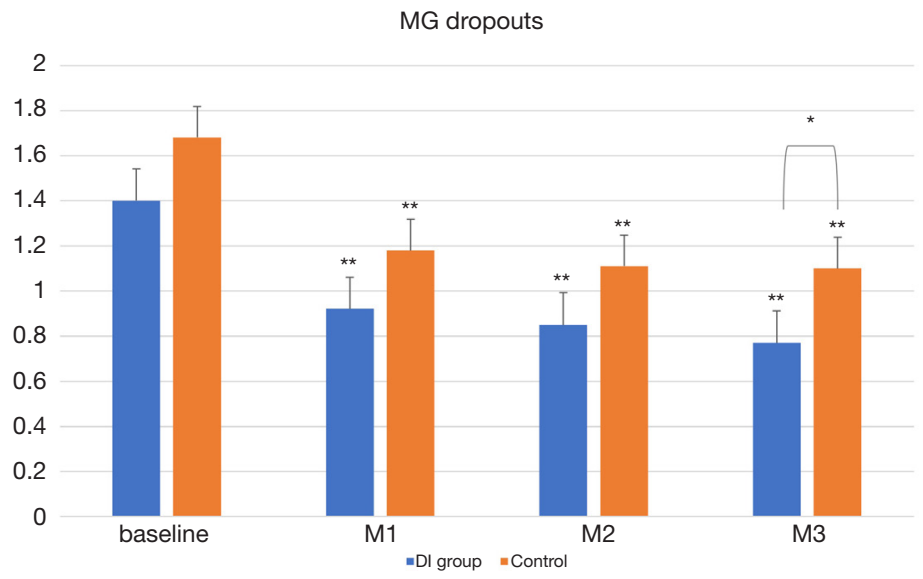

Figure $4 \mathrm{MG}$ dropouts at baseline, M1, M2, and M3 in the DI group and control group (orange). Both groups showed significant improvement from baseline at any visit $(\mathrm{P}<0.01)$. The DI group showed better improvement than the control group at $\mathrm{M} 3$ ( $\mathrm{P}<0.05)$. ${ }^{*} \mathrm{P}<0.05 ;{ }^{* *} \mathrm{P}<0.01$. M1, month $1 ; \mathrm{M} 2$, month2; M3, month 3; DI, Demodex infestation.

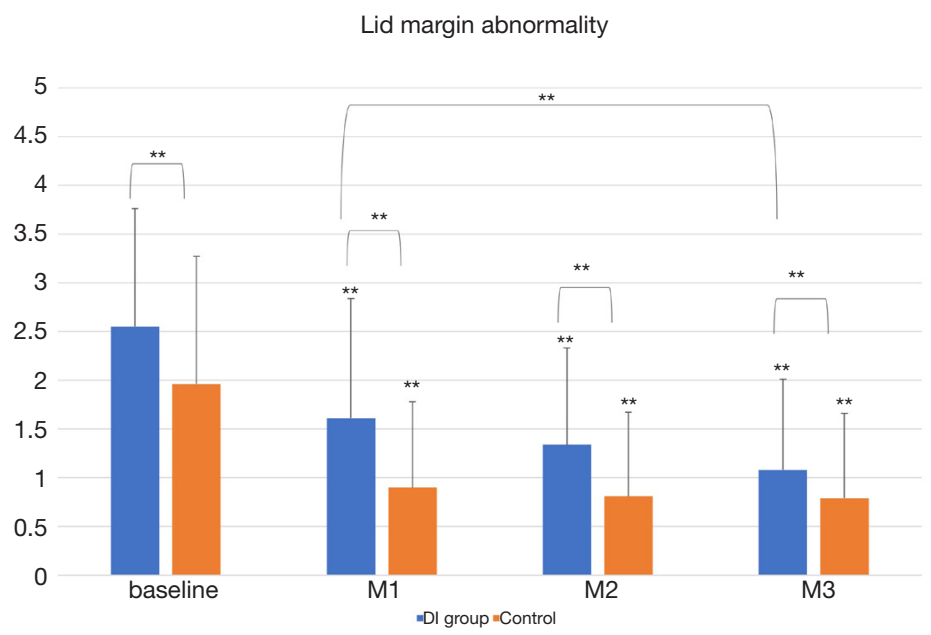

Figure 5 Lid margin abnormality at baseline, M1, M2, and M3 in the DI group and control group. There was a significant difference between the DI group and control group at any time point (all, $\mathrm{P}<0.01$ ). In the DI group, the improvement from $\mathrm{M} 1$ to $\mathrm{M} 3$ was significantly different $(\mathrm{P}<0.01)$. Lid margin abnormality in both groups was improved significantly from baseline at any visit. ${ }^{* *} \mathrm{P}<0.01$. M1, month $1 ; \mathrm{M} 2$, month 2; M3, month 3; DI, Demodex infestation.

present study, the eradication rate improved from $59.5 \%$ at $\mathrm{M} 1$ to $66.67 \%$ at $\mathrm{M} 2$, and reached $83.3 \%$ at M3. Our results are similar to those of Zhang et al. in that the eradication rate continuously improved steadily 3 months after OPT treatment. We speculate that the OPT treatment may affect the reproductive capacity of the mites.

The mechanism of inducing Demodex coagulation necrosis while preserving the surrounding follicle is curious. In vitro experiments have shown that the optimal temperature for Demodex development is $16-20{ }^{\circ} \mathrm{C}$.
Temperatures of $<0$ or $>37^{\circ} \mathrm{C}$ are harmful to the mites. Lethal temperature is $54{ }^{\circ} \mathrm{C}$, and the effective killing temperature is $58{ }^{\circ} \mathrm{C}(25,28)$. The fifth-generation M22 system uses an OPT model that can increase the temperature in the targeted tissue area to a maximum of $70{ }^{\circ} \mathrm{C}$ without epidermal burning (29,30). We considered the high temperature generated by the OPT as the lethal temperature for the mites. Prieto et al. suggested that the mites contain a chromophore that renders them more sensitive to absorbing the energy delivered by the OPT. 
It is likely that the approximately spherical structure of the mites cannot transfer energy as much as the openended cylindrical hair follicles (8). Moreover, Zhang et al. suggested that IPL may affect the germ of the mites to inhibit their reproductive ability, and the high temperature may damage the environment in which the mites live (9).

In addition to the therapeutic effect in eradicating Demodex mites, we found that OPT treatment improved the symptoms and signs of blepharitis and MGD. Meibum composition, MG structure, and subjective symptom scores improved significantly from baseline to post-treatment in both the groups. Our results are in agreement with several trials that have demonstrated the efficacy of IPL in treating MGD (31,32). The mechanism by which these effects are manifested still remains unclear, although there are several hypotheses such as stimulating the release of inflammatory mediators or softening the sticky meibum as a result of heat transfer to the eyelids and MGs. Our study confirms the above hypotheses. From our point of view, killing Demodex mites would have the direct effect of improving the symptoms of anterior and posterior blepharitis; the latter may be related to the death of D. brevis in the MGs. The indirect effect could be related to the decrease additional bacteria and fungi, or to the host's immune response.

There were several limitations to our study. As the benefits of OPT treatment may be cumulative, a longterm study is necessary to better understand the effect and mechanism of Demodex eradication by OPT. In addition, although Demodex infestation is classically diagnosed by analyzing depilated eyelashes under a light microscope, this method cannot detect the presence of D. brevis inside MGs. IVCM is more accurate for detecting the presence of mites inside follicles or MGs, and for assessing the MG microstructure. Therefore, similar studies using IVCM will be required for the accurate detection of these mites.

\section{Conclusions}

It is the first prospective, consecutive, controlled study on ocular Demodex infestation using a large sample size. Our findings suggest that in patients with MGD, Demodex infestation may cause more ocular surface damage and inflammation, less efficiency of lid margin abnormality, and slower recovery of corneal staining and conjunctival congestion after OPT treatment compared with that in those without Demodex infestation.

\section{Acknowledgments}

We thank Yujie Mou (Department of Ophthalmology, The Second Affiliated Hospital of Zhejiang University School of Medicine, China) for her support in the statistical work of this study.

Funding: This work was supported by the Zhejiang Natural Science Foundation [LY20H120009]; the Natural Science Foundation of China [31751003]; the Key Research and Development Project of Zhejiang Province [2020C03035]; and the Major Science and Technology Projects of Zhejiang Province [2017C03046].

\section{Footnote}

Reporting Checklist: All authors have completed the STROBE reporting checklist. Available at http://dx.doi. org/10.21037/atm-20-1745

Data Sharing Statement: Available at http://dx.doi. org/10.21037/atm-20-1745

Conflicts of Interest: All authors have completed the ICMJE uniform disclosure form (available at http://dx.doi. org/10.21037/atm-20-1745). The authors have no conflicts of interest or financial disclosures to declare.

Ethical Statement: The authors are accountable for all aspects of the work in ensuring that questions related to the accuracy or integrity of any part of the work are appropriately investigated and resolved. The study was conducted in accordance with the Declaration of Helsinki (as revised in 2013) and was approved by the Ethics Committee of the Second Affiliated Hospital of Zhejiang University School of Medicine (No. 2019-283). Informed consent was taken from all the patients.

Open Access Statement: This is an Open Access article distributed in accordance with the Creative Commons Attribution-NonCommercial-NoDerivs 4.0 International License (CC BY-NC-ND 4.0), which permits the noncommercial replication and distribution of the article with the strict proviso that no changes or edits are made and the original work is properly cited (including links to both the formal publication through the relevant DOI and the license). See: https://creativecommons.org/licenses/by-nc-nd/4.0/. 


\section{References}

1. English FP, Nutting WB. Demodicosis of ophthalmic concern. Am J Ophthalmol 1981;91:362-72.

2. Kheirkhah A, Casas V, Li W, et al. Ocular manifestations of ocular demodex infestaion. Am J Ophthalmol 2007;143:743-9.

3. Liang L, Safran S, Gao Y, et al. Ocular demodicosis as a potential cause of pediatric blepharoconjunctivitis. Cornea 2010;29:1386-91.

4. Raulin C, Goldman MP, Weiss MA, et al. Treatment of adult port-wine stains using intense pulsed light therapy (PhotoDerm VL): brief initial clinical report. Dermatol Surg 1997;23:594-7.

5. Jiang X, Lv H, Song H, et al. Evaluation of the safety and effectiveness of intense pulsed light in the treatment of meibomian gland dysfunction. J Ophthalmol 2016;2016:1910694.

6. Gupta PK, Vora GK, Matossian C, et al. Outcomes of intense pulsed light therapy for treatment of evaporative dry eye disease. Can J Ophthalmol 2016;51:249-53.

7. Yan X, Hong J, Jin X, et al. The efficacy of intense pulsed combined with meibomian gland expression for the treatment of dry eye disease due to meibomian gland dysfunction: a multicenter, randomized controlled trial. Eye Contact Lens 2021;47:45-53.

8. Prieto VG, Sadick N, Lloreta J, et al. Effects of intense pulsed light on sun-damaged human skin, routine, and ultrastructural analysis. Lasers Surg Med 2002;30:82-5.

9. Zhang X, Song N, Gong L. Therapeutic Effect of Intense Pulsed Light on Ocular Demodicosis. Curr Eye Res 2019;44:250-6.

10. Cheng SN, Jiang FG, Chen H, et al. Intense Pulsed Light Therapy for Patients with Meibomian Gland Dysfunction and Ocular Demodex Infestation. Curr Med Sci 2019;39:800-9.

11. Fitzpatrick T B. The validity and practicality of sunreactive skin types I through VI. Arch Dermatol 1988;124:869-71.

12. Gao YY, Pascuale MA, Li W, et al. High prevalence of Demodex in eyelashes with cylindrical dandruff. Invest Ophthalmol Vis Sci 2005;46:3089-94.

13. Nelson J D, Shimazaki J, Benitez-del-Castillo, et al. The international workshop on meibomian gland dysfunction: report of the definition and classification subcommittee. Invest Ophthalmol Vis Sci 2011;52:1930-7.

14. Jie Y, Xu L, Wu YY, et al. Prevalence of dry eye among adult Chinese in the Beijing Eye Study. Eye (Lond)
2009;23:688-93.

15. Ding J, Sullivan D A. Aging and dry eye disease. Exp Gerontol 2012;47:483-90.

16. Bhandari $\mathrm{V}$ and Reddy J K. Blepharitis: always remember demodex. Middle East Afr J Ophthalmol 2014;21:317-20.

17. Chen D, Li R, Liu X W, et al. Prevalence and treatment effects of Demodex species in eyelash follicles in patients with meibomian gland dysfunction. Zhonghua Yan Ke Za Zhi 2017;53:193-7.

18. Kabatas N, Dogan A S, Kabatas E U, et al. The Effect of Demodex Infestation on Blepharitis and the Ocular Symptoms. Eye Contact Lens 2017;43:64-7.

19. Junk A K, Lukacs A, Kampik A. Topical administration of metronidazole gel as an effective therapy alternative in chronic Demodex blepharitis--a case report. Klin Monbl Augenheilkd 1998;213:48-50.

20. Gao YY, Di Pascuale MA, Li W, et al. In vitro and in vivo killing of ocular Demodex by tea tree oil. Br J Ophthalmol 2005;89:1468-73.

21. Fulk GW, Murphy B, Robins MD. Pilocarpine gel for the treatment of demodicosis--a case series. Optom Vis Sci 1996;73:742-5.

22. Rodriguez AE, Ferrer C, Alio JL. Chronic blepharitis and Demodex. Arch Soc Esp Oftalmol 2005;80:635-42.

23. Salem DA, El-Shazly A, Nabih N, et al. Evaluation of the efficacy of oral ivermectin in comparison with ivermectinmetronidazole combined therapy in the treatment of ocular and skin lesions of Demodex folliculorum. Int J Infect Dis 2013;17:e343-47.

24. Jansen T, Kastner U, Kreuter A, et al. Rosacea-like demodicidosis associated with acquired immunodeficiency syndrome. Br J Dermatol 2001;144:139-42.

25. Murube J. Demodex hominis. Ocul Surf 2015;13:181-6.

26. Zhao YE, Wu LP, Hu L, et al. Association of blepharitis with Demodex: a meta-analysis. Ophthalmic Epidemiol 2012;19:95-102.

27. Randon $M$, Liang $H$, Hamdaoui $M$, et al. In vivo confocal microscopy as a novel and reliable tool for the diagnosis of Demodex eyelid infestation. Br J Ophthalmol 2015;99:336-41.

28. Zhao YE, Guo N, Wu LP. The effect of temperature on the viability of Demodex folliculorum and Demodex brevis. Parasitol Res 2009;105:1623-8.

29. Raulin C, Greve B, Grema H. IPL technology: a review. Lasers Surg Med 2003;32:78-87.

30. VuralE, Winfield HL, Shingleton AW, et al. The effects of laser irradiation on Trichophyton rubrum growth. Lasers Med Sci 2008;23:349-53.

31. Craig JP, Chen YH, Turnbul PRK. Prospective trial of 
Page 12 of 12

intense pulsed light for the treatment of meibomian gland dysfunction. Invest Ophthalmol Vis Sci 2015;56:1965-70.

32. Dell SJ, Gaster RN, Barbarino SC, et al. Prospective evaluation of intense pulsed light and meibomian gland

Cite this article as: Huo Y, Mo Y, Wu Y, Fang F, Jin X. Therapeutic effect of intense pulsed light with optimal pulse technology on meibomian gland dysfunction with and without ocular Demodex infestation. Ann Transl Med 2021;9(3):238. doi: 10.21037/atm-20-1745
Huo et al. OPT + MGX for Demodex in meibomian gland dysfunction

expression efficacy on relieving signs and symptoms of dry eye disease due to meibomian gland dysfunction. Clin Ophthalmol 2017;11:817-27. 